\title{
Synthesis and Spectroscopic Properties of a Hexapyrenylbenzene Derivative
}

Dirk Rausch, Christoph Lambert*

Institut für organsiche Chemie, Julius-Maximilians-Universität, Am Hubland, D-97074

Würzburg, Germany

\section{Supporting Information}

General: Reagents and solvents were purchased from Acros, Aldrich, and Fluka and were used as received. 4,5,9,10-tetrahydropyrene was synthesized according to a literature procedure ${ }^{1}$. Dioxane and THF were distilled from $\mathrm{Na}$ /benzophenone. Benzene was distilled from $\mathrm{CaH}_{2}$. All reactions except the first were carried out under a dry $\mathrm{N}_{2}$ atmosphere. ${ }^{1} \mathrm{H}$ and ${ }^{13} \mathrm{C}$ NMR spectra were measured on a Bruker $400 \mathrm{MHz}$ spectrometer. Chemical shifts are reported in ppm downfield from $\mathrm{SiMe}_{4}$, using the solvent's residual signal as an internal reference. Coupling constants $(\mathrm{J})$ are given in $\mathrm{Hz}$. Resonance multiplicities are described as $\mathrm{s}$ (singlet), $\mathrm{d}$ (doublet), $\mathrm{t}$ (triplet), or $\mathrm{m}$ (multiplet). MALDI mass spectra were obtained on a Bruker Daltonics Autoflex II instrument using DCTB as the matrix.

\section{2,7-Dibromo-4,5,9,10-tetrahydropyrene.}<smiles>Brc1cc2c3c(c1)CCc1cc(Br)cc(c1-3)CC2</smiles>

To a stirred solution of 4,5,9,10-tetrahydropyrene $(4.00 \mathrm{~g}, 19.4 \mathrm{mmol})$ in $130 \mathrm{ml}$ acetic acid a solution of sodium hydroxide $(1.71 \mathrm{~g}, 42.7 \mathrm{mmol})$ and bromine $(6.82 \mathrm{~g}$, $2.19 \mathrm{ml}, 42.7 \mathrm{mmol}$ ) in $130 \mathrm{ml}$ water was added dropwise over a periode of $30 \mathrm{~min}$ at ambient temperature. The reaction mixture was stirred overnight and the precipitate was removed by filtration, washed with water and dried in vacuo. The white solid was recrystallized from benzene to give 2,7-dibromo-4,5,9,10-tetrahydropyrene (3.96 g, $10.8 \mathrm{mmol}, 56 \%$ ). Mp: $218-219{ }^{\circ} \mathrm{C} .{ }^{1} \mathrm{H}$ NMR $\left(400 \mathrm{MHz}, \mathrm{CDCl}_{3}\right): \delta 2.82(\mathrm{~s}, 8 \mathrm{H}), 7.20$ (s, 4H). CA: [17533-36-7]. 


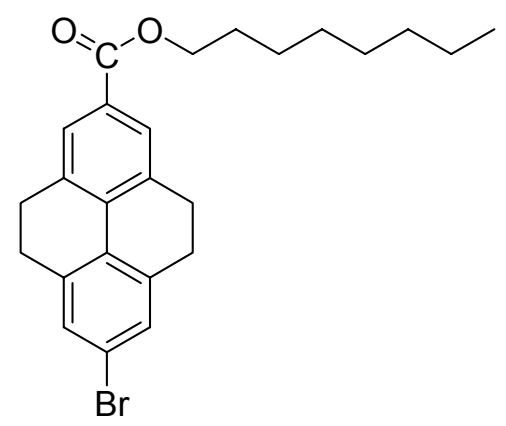

To a solution of 2,7-dibromo-4,5,9,10-tetrahydropyrene (1.08 g, $2.97 \mathrm{mmol})$ in $100 \mathrm{ml}$ tetrahydrofuran, $n$-butyllithium solution in hexane $(1.6 \mathrm{M}, 1.85 \mathrm{ml}, 2.97 \mathrm{mmol})$ was added dropwise during $5 \mathrm{~min}$ at $-90{ }^{\circ} \mathrm{C}$ and stirred for $25 \mathrm{~min}$. Then octylchloroformate $(1.71 \mathrm{~g}, 1.74 \mathrm{ml}, 8.90 \mathrm{mmol})$ was added in one portion and stirring was continued for further $15 \mathrm{~min}$. The reaction mixture was poured on crushed ice and some drops conc. hydrochloric acid and was extracted three times with dichloromethane. The organic phase was dried with $\mathrm{MgSO}_{4}$ and evaporated. The residue was purified by flash chromatography on silica gel using petroleum ether/ethyl acetate $98: 2$ as eluent to yield 3 as a white solid $(0.95 \mathrm{~g}, 2.15 \mathrm{mmol}, 73$ \%). Mp: $103{ }^{\circ} \mathrm{C} .{ }^{1} \mathrm{H}$ NMR (400 MHz, $\left.\mathrm{CDCl}_{3}\right): \delta 0.89$ (t, 3H), 1.2-1.5 (10H), $1.78(\mathrm{~m}$, $2 \mathrm{H}), 2.89(\mathrm{~m}, 8 \mathrm{H}), 4.32(\mathrm{t}, 3 \mathrm{H}), 7.25(\mathrm{~s}, 2 \mathrm{H}), 7.75(\mathrm{~s}, 2 \mathrm{H}) .{ }^{13} \mathrm{C} \mathrm{NMR}(400 \mathrm{MHz}$, $\left.\mathrm{CDCl}_{3}\right): \delta 14.2\left(\mathrm{CH}_{3}\right), 22.8\left(\mathrm{CH}_{2}\right), 26.2\left(\mathrm{CH}_{2}\right), 28.08\left(\mathrm{CH}_{2}\right), 28.10\left(\mathrm{CH}_{2}\right), 28.9\left(\mathrm{CH}_{2}\right)$, $29.3\left(\mathrm{CH}_{2}\right), 29.4\left(\mathrm{CH}_{2}\right), 32.0\left(\mathrm{CH}_{2}\right), 65.3\left(\mathrm{CH}_{2}\right), 121.9(\mathrm{q}), 127.4(\mathrm{CH}), 129.0(\mathrm{q})$, $129.2(\mathrm{q}+\mathrm{CH}), 134.3(\mathrm{q}), 135.2(\mathrm{q}), 138.3(\mathrm{q}), 166.9(\mathrm{C}(\mathrm{O})=\mathrm{O}) . \mathrm{MS}(\mathrm{El}): \mathrm{m} / \mathrm{z}(\%)$, $440.2(100)$ 


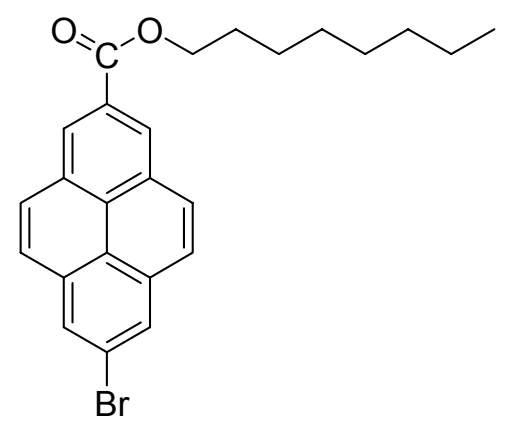

A solution of $3(760 \mathrm{mg}, 1.72 \mathrm{mmol})$ and DDQ $(821 \mathrm{mg}, 3.62 \mathrm{mmol})$ in dry benzene was refluxed for 24 - $48 \mathrm{~h}$ under nitrogen atmosphere (reaction status controlled by TLC). After removal of the solvent the residue was treated with cold ethanol to give a white solid which was filtered off (470 mg, $1.08 \mathrm{mmol}, 62 \%)$. Mp: $131-132{ }^{\circ} \mathrm{C} .{ }^{1} \mathrm{H}$ NMR (400 MHz, $\left.\mathrm{CDCl}_{3}\right): \delta 0.90(\mathrm{t}, 3 \mathrm{H}), 1.2-1.5(10 \mathrm{H}), 1.90(\mathrm{~m}, 2 \mathrm{H}), 4.49(\mathrm{t}, 2 \mathrm{H}), 8.02$ $\left(\mathrm{AA}^{\prime}, 2 \mathrm{H}\right), 8.16\left(\mathrm{BB}^{\prime}, 2 \mathrm{H}\right), 8.32(\mathrm{~s}, 2 \mathrm{H}), 8.85(\mathrm{~s}, 2 \mathrm{H}) .{ }^{13} \mathrm{C} \mathrm{NMR}\left(400 \mathrm{MHz}, \mathrm{CDCl}_{3}\right): \delta$ $14.3\left(\mathrm{CH}_{3}\right), 22.8\left(\mathrm{CH}_{2}\right), 26.3\left(\mathrm{CH}_{3}\right), 29.0\left(\mathrm{CH}_{2}\right), 29.4\left(\mathrm{CH}_{2}\right), 29.5\left(\mathrm{CH}_{2}\right), 32.0\left(\mathrm{CH}_{2}\right)$, $65.8\left(\mathrm{CH}_{2}\right), 121.4(\mathrm{q}), 123.1(\mathrm{q}), 126.6(\mathrm{CH}), 126.7(\mathrm{q}), 127.2(\mathrm{CH}), 127.7(\mathrm{CH}), 128.2$ (q), $129.2(\mathrm{CH}), 130.8$ (q), 133.4 (q), $167.2(\mathrm{C}(\mathrm{O})=\mathrm{O})$. MS (EI): m/z (\%), 436.3 (100). HRMS (El): calcd. $\mathrm{m} / \mathrm{z}=436.10388$, found $\mathrm{m} / \mathrm{z}=436.10333$.

7-(Trimethylsilylethynyl)-2-pyrenecarboxylic acid octylester (5).

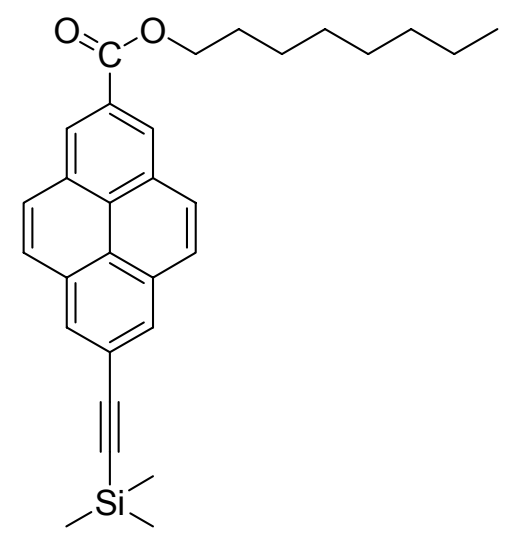

Compound 4 (300 mg, $0.69 \mathrm{mmol}$ ) was dissolved in $7 \mathrm{ml}$ dioxane (slightly heating required) under nitrogen atmosphere. To this solution copper(I)iodide (3.20 mg, 17.1 $\mu \mathrm{mol}), \quad$ bis(benzonitrile)palladium(II)chloride $\quad(7.90 \mathrm{mg}, \quad 20.6 \mu \mathrm{mol}), \quad$ tri-tert- 
butylphosphine solution in hexane $(0.33 \mathrm{M}, 0.21 \mathrm{ml}, 68.8 \mu \mathrm{mol})$, trimethylsilylacetylene $(84.2 \mathrm{mg}, 121 \mu \mathrm{l}, 0.86 \mathrm{mmol})$ and diisopropylamine $(86.7 \mathrm{mg}$, $121 \mu \mathrm{l}, 0.86 \mathrm{mmol}$ ) were added. The mixture was stirred at room temperature overnight. The solvent was removed in vacuo and the residue extracted with dichloromethane and water. After drying the organic phase with $\mathrm{MgSO}_{4}$ the dichloromethane was evaporated and the residue was purified by flash chromatography on silica gel using petroleum ether/ethyl acetate 95:5 as eluent to yield 5 (220 mg, $0.48 \mathrm{mmol}, 70 \%$ ) which still contains minor impurities. Mp: 120-122 ${ }^{\circ} \mathrm{C} .{ }^{1} \mathrm{H}$ NMR $\left(400 \mathrm{MHz}, \mathrm{CDCl}_{3}\right): \delta 0.34(\mathrm{~s}, 9 \mathrm{H}), 0.90(\mathrm{t}, 3 \mathrm{H}), 1.2-1.5(10 \mathrm{H}), 1.90(\mathrm{~m}$, $2 \mathrm{H}), 4.48$ (t, 2H), $8.04\left(\mathrm{AA}^{\prime}, 2 \mathrm{H}\right), 8.14$ (BB', 2H), 8.30 (s, 2H), 8.83 (s, 2H). MS (El): m/z (\%), 454.2 (100), 439.2 (15). HRMS (El): calcd. $\mathrm{m} / \mathrm{z}=454.23287$, found $\mathrm{m} / \mathrm{z}=$ 454,23232

\section{7-Ethynyl-2-pyrenecarboxylic acid octylester (6).}

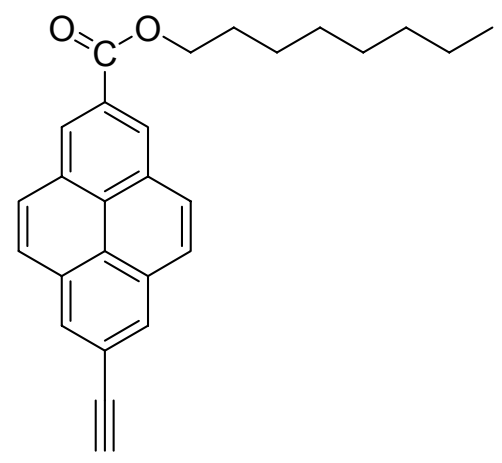

A solution of $5(211 \mathrm{mg}, 0.46 \mathrm{mmol})$ in $5 \mathrm{ml}$ tetrahydrofuran was treated with tetrabutylammonium fluoride solution $(1 \mathrm{M}, 0.51 \mathrm{ml}, 0.51 \mathrm{mmol})$ in tetrahydrofuran. After $2 \mathrm{~h}$ the solvent was removed in vacuo and the residue extracted with dichloromethane and water. The organic phase was dried with $\mathrm{MgSO}_{4}$ and evaporated to dryness to yield $170 \mathrm{mg}(0.45 \mathrm{mmol}, 96 \%)$. The crude product was used without further purification. Mp: $98^{\circ} \mathrm{C}$. ${ }^{1} \mathrm{H}$ NMR $\left(400 \mathrm{MHz}, \mathrm{CDCl}_{3}\right): \delta 0.90(\mathrm{t}, 3 \mathrm{H})$, 1.2-1.5 (10H), $1.90(\mathrm{~m}, 2 \mathrm{H}), 3.27(\mathrm{~s}, 1 \mathrm{H}), 4.48$ (t, 2H), 8.06 (AA', 2H), 8.16 (BB', 2H), $8.32(\mathrm{~s}, 2 \mathrm{H}), 8.85(\mathrm{~s}, 2 \mathrm{H})$. 


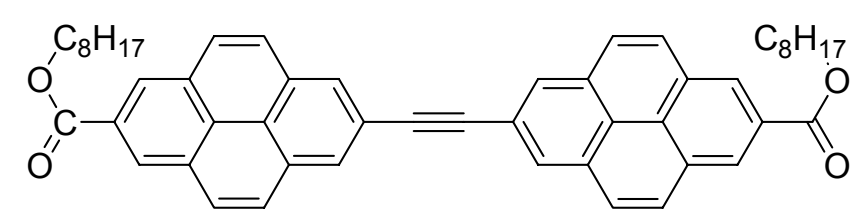

Compound $4(72.0 \mathrm{mg}, 16.5 \mu \mathrm{mol})$ was dissolved in $3 \mathrm{ml}$ dioxane (slightly heating required) under nitrogen atmosphere. To the solution copper(I)iodide ( $3.15 \mathrm{mg}, 16.5$ $\mu \mathrm{mol}), \quad$ bis(benzonitrile)palladium(II)chloride $\quad(3.35 \mathrm{mg}, \quad 8.27 \mu \mathrm{mol}), \quad$ tri-tertbutylphosphine solution in hexane $(0.33 \mathrm{M}, 0.05 \mathrm{ml}, 16.5 \mu \mathrm{mol}), 6(70.0 \mathrm{mg}, 18.2$ $\mu \mathrm{mol})$ and diisopropylamine $(20.1 \mathrm{mg}, 27.8 \mu \mathrm{l}, 0.86 \mathrm{mmol})$ were added. The mixture was stirred at room temperature overnight. The solvent was removed in vacuo and the residue extracted with dichloromethane and water. After drying the organic phase with $\mathrm{MgSO}_{4}$ the dichloromethane was removed and the residue was washed several times with cold dichloromethane to yield 7 (63.0 mg, $85.3 \mu \mathrm{mol}, 52 \%$ ). Mp: 263-267 ${ }^{\circ} \mathrm{C} .{ }^{1} \mathrm{H}$ NMR $\left(400 \mathrm{MHz}, \mathrm{CDCl}_{3}\right): \delta 0.91(\mathrm{t}, 6 \mathrm{H}), 1.2-1.5(2 \mathrm{H}), 1.91(\mathrm{~m}, 4 \mathrm{H}), 4.49(\mathrm{t}$, $4 \mathrm{H}), 8.12\left(\mathrm{AA}^{\prime}, 4 \mathrm{H}\right), 8.18\left(\mathrm{BB}^{\prime}, 4 \mathrm{H}\right), 8.46(\mathrm{~s}, 4 \mathrm{H}), 8.48(\mathrm{~s}, 4 \mathrm{H}) . \mathrm{MS}(\mathrm{El}): \mathrm{m} / \mathrm{z}(\%)$, 738.3 (100), 626.2 (10). HRMS (EI): calcd. $\mathrm{m} / \mathrm{z}=738.37164$, found $=738.37109$ 
Hexa-7-(2-pyrenylcarboxylic acid, octyl ester)benzene (8).

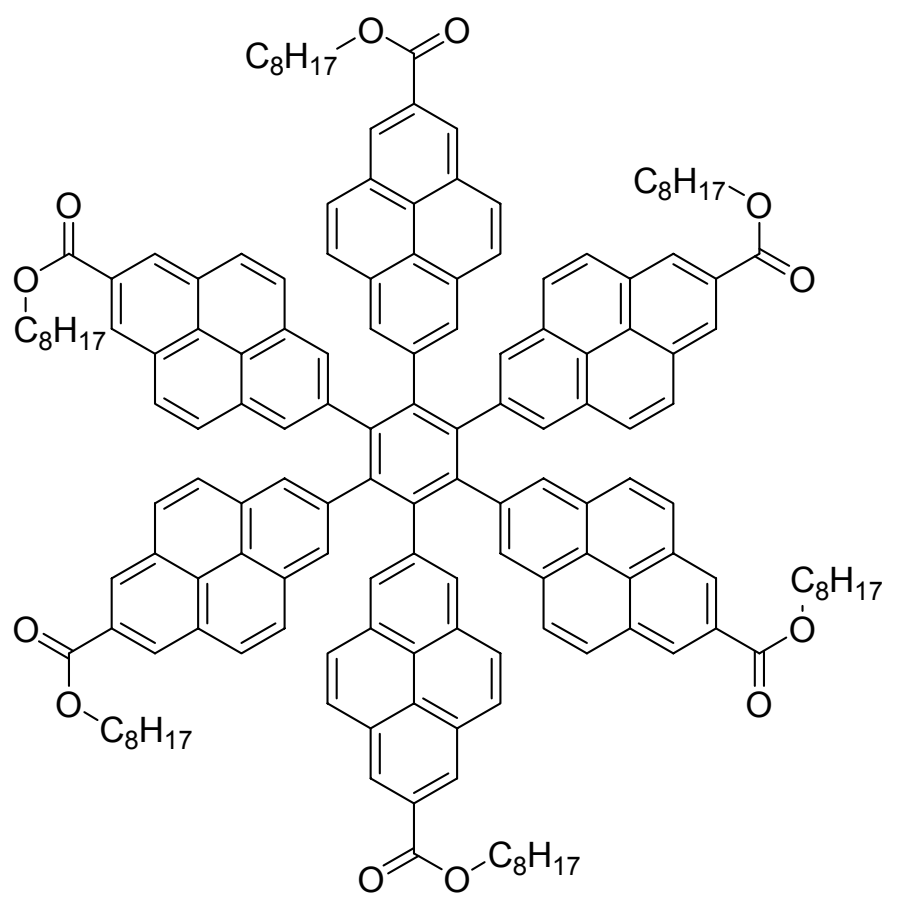

A mixture of $7(100 \mathrm{mg}, 0.14 \mathrm{mmol})$ and $25 \mathrm{ml}$ dioxane was heated to $60^{\circ} \mathrm{C}$ under nitrogen. Then $\mathrm{Co}_{2} \mathrm{CO}_{8}(15.0 \mathrm{mg}, 45.0 \mu \mathrm{mol})$ was added and the mixture was heated to reflux overnight. The solvent was removed in vacuo and the residue was purified by flash-chromatography on silica gel using petrol ether/dichloromethane 2:1 -> 3:1 as eluent. The crude product was precipitated twice from dichloromethane and methanol to yield pure pale yellow 8 ( $25 \mathrm{mg}, 35 \mathrm{~mol}$ ). $\mathrm{Mp}: 419{ }^{\circ} \mathrm{C}$ (decomp.). ${ }^{1} \mathrm{H}$ NMR (400 MHz, $\left.\mathrm{CDCl}_{3}\right): 0.83(\mathrm{t}, 18 \mathrm{H}), 1.2-1.5(60 \mathrm{H}), 1.73(\mathrm{~m}, 12 \mathrm{H}), 4.30(\mathrm{t}, 12 \mathrm{H})$, 7.55 (AA', 12H), 7.64 (BB', 12H). 8.08 (s, 12H), 8.43 (s, 12H). ${ }^{13} \mathrm{C} \mathrm{NMR}(400 \mathrm{MHz}$ $\left.\mathrm{CDCl}_{3}\right)$ : $14.2\left(\mathrm{CH}_{3}\right), 22.7\left(\mathrm{CH}_{2}\right), 26.1\left(\mathrm{CH}_{2}\right), 28.8\left(\mathrm{CH}_{2}\right), 29.27\left(\mathrm{CH}_{2}\right), 29.34\left(\mathrm{CH}_{2}\right)$, $31.9\left(\mathrm{CH}_{2}\right), 65.5\left(\mathrm{CH}_{2}\right), 122.2(\mathrm{q}), 125.4(\mathrm{CH}), 126.3(\mathrm{q}), 127.2(\mathrm{q}), 127.6(2 \times \mathrm{CH})$, 128.3 (q), 130.5 (q), 130.6 (q), 139.1 (q), 141.9 (q), 167.2 (C(O)=O). MS (MALDI), $\mathrm{m} / \mathrm{z}(\%) 2216.039$ (100), calcd. 2216.11609 (100). 
DFT calculations (B3LYP/6-31G*) of pyrene LUMO and HOMO

Pyrene LUMO

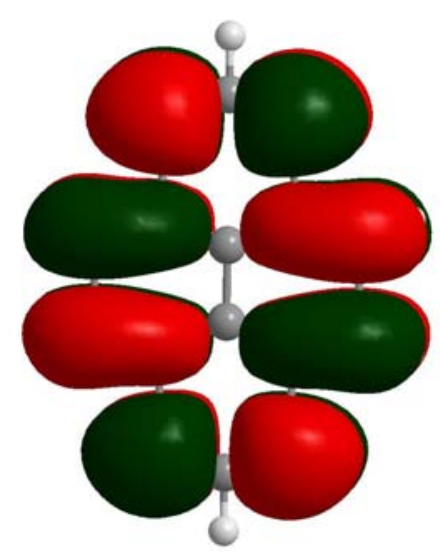

\section{Pyrene HOMO}

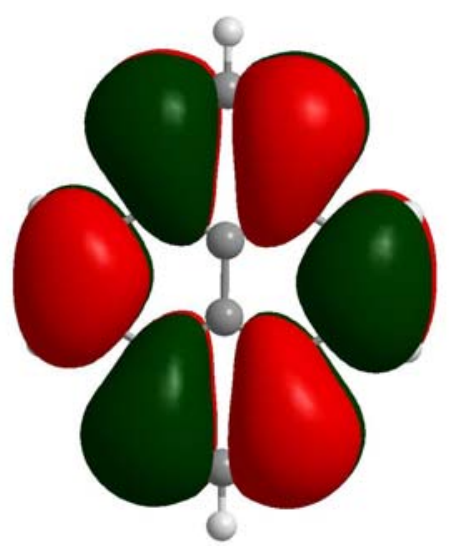


${ }^{13} \mathrm{C} \mathrm{NMR}\left(\mathrm{CDCl}_{3}\right)$ of 3

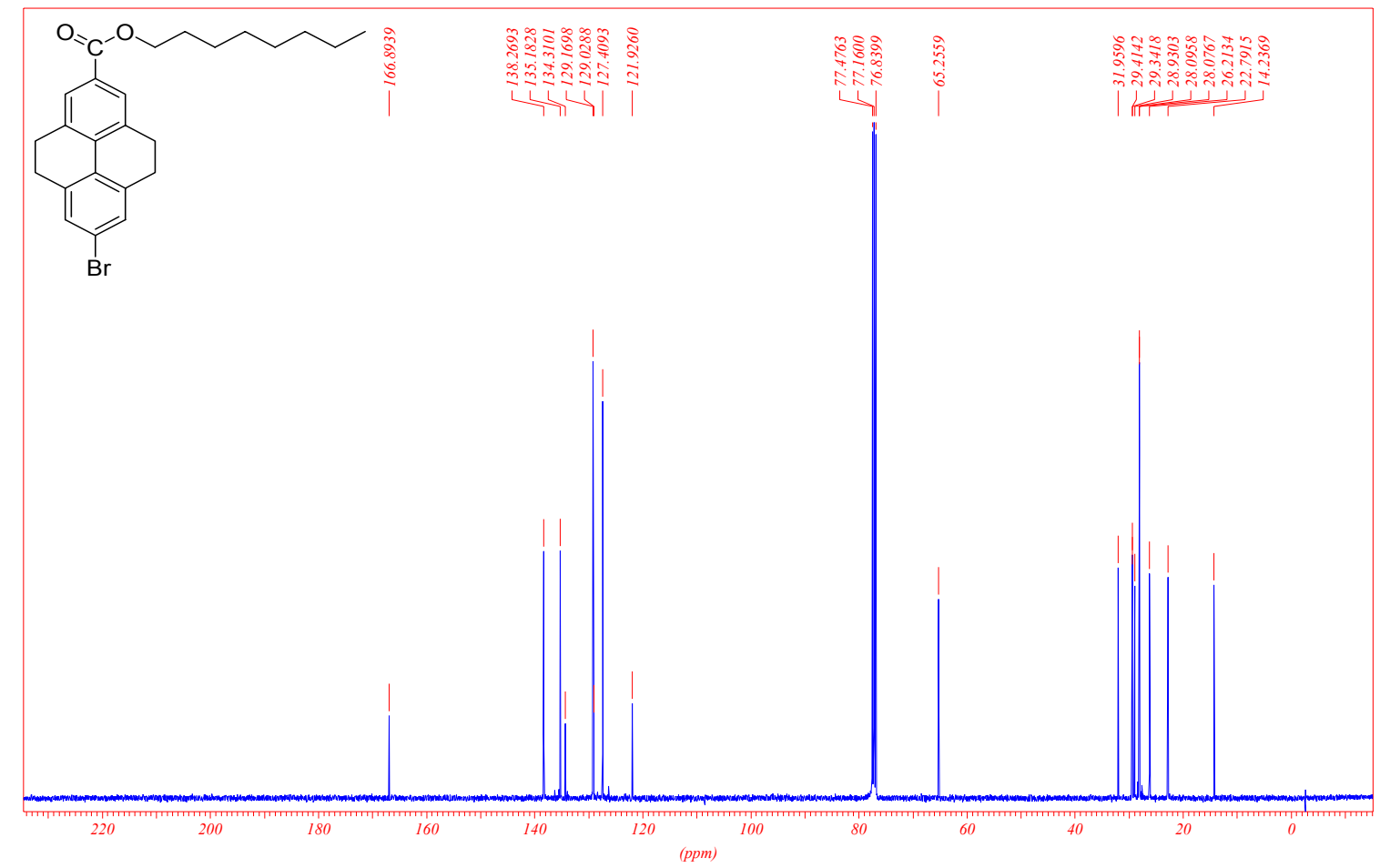

\section{${ }^{1} \mathrm{H}$ NMR $\left(\mathrm{CDCl}_{3}\right)$ of $\mathbf{3}$}

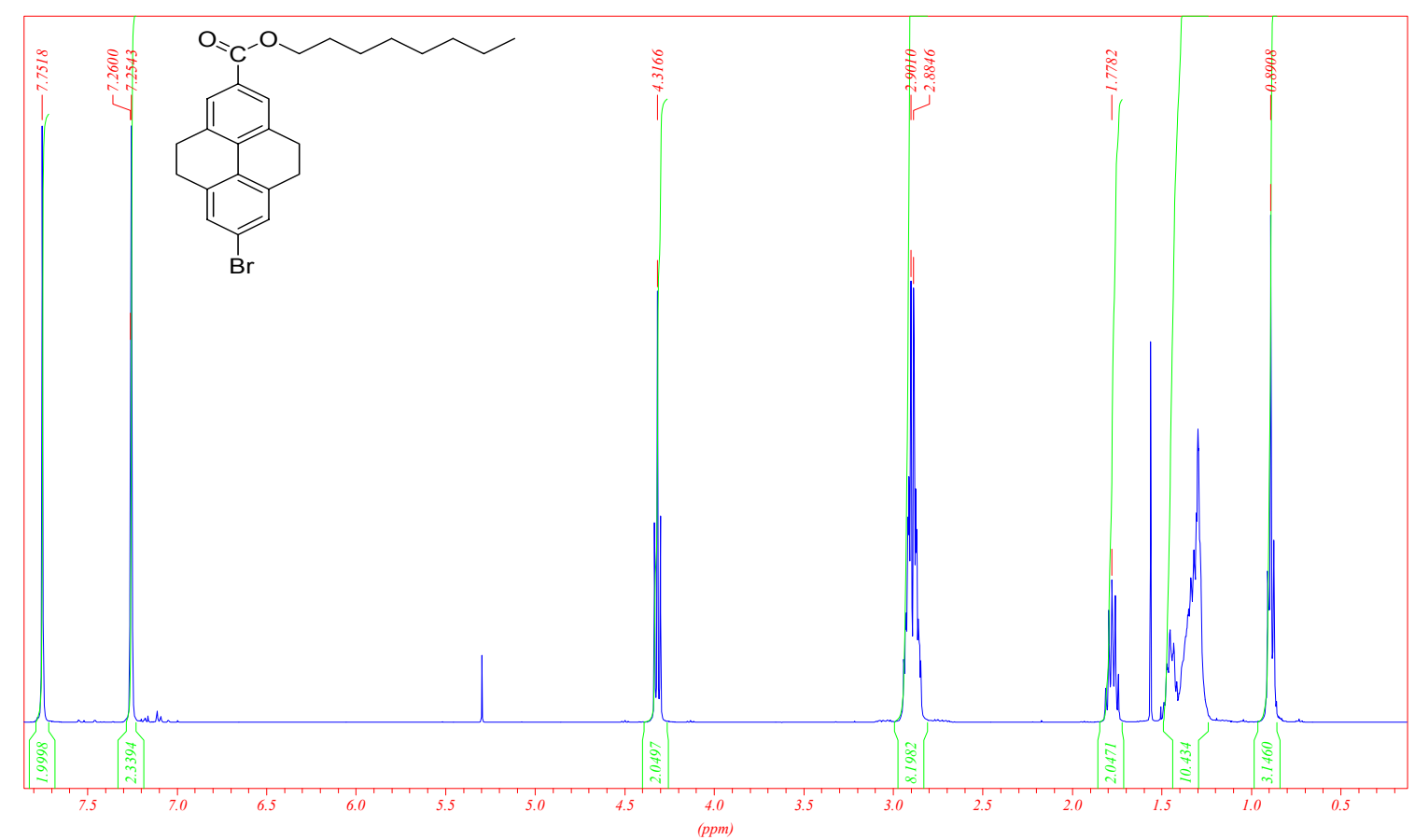


${ }^{13} \mathrm{C}$ NMR $\left(\mathrm{CDCl}_{3}\right)$ of 4

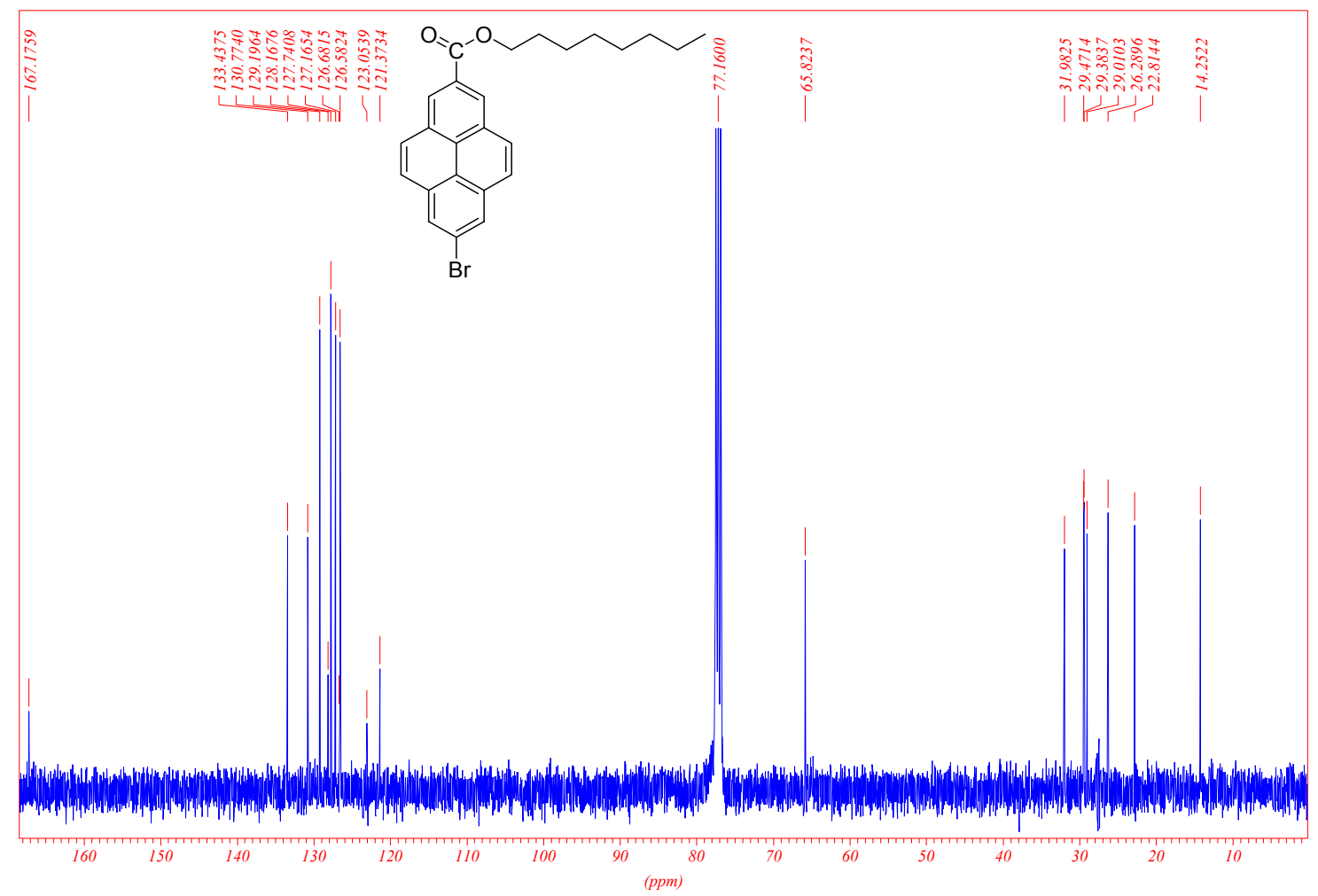

${ }^{1} \mathrm{H}$ NMR $\left(\mathrm{CDCl}_{3}\right)$ of $\mathbf{4}$

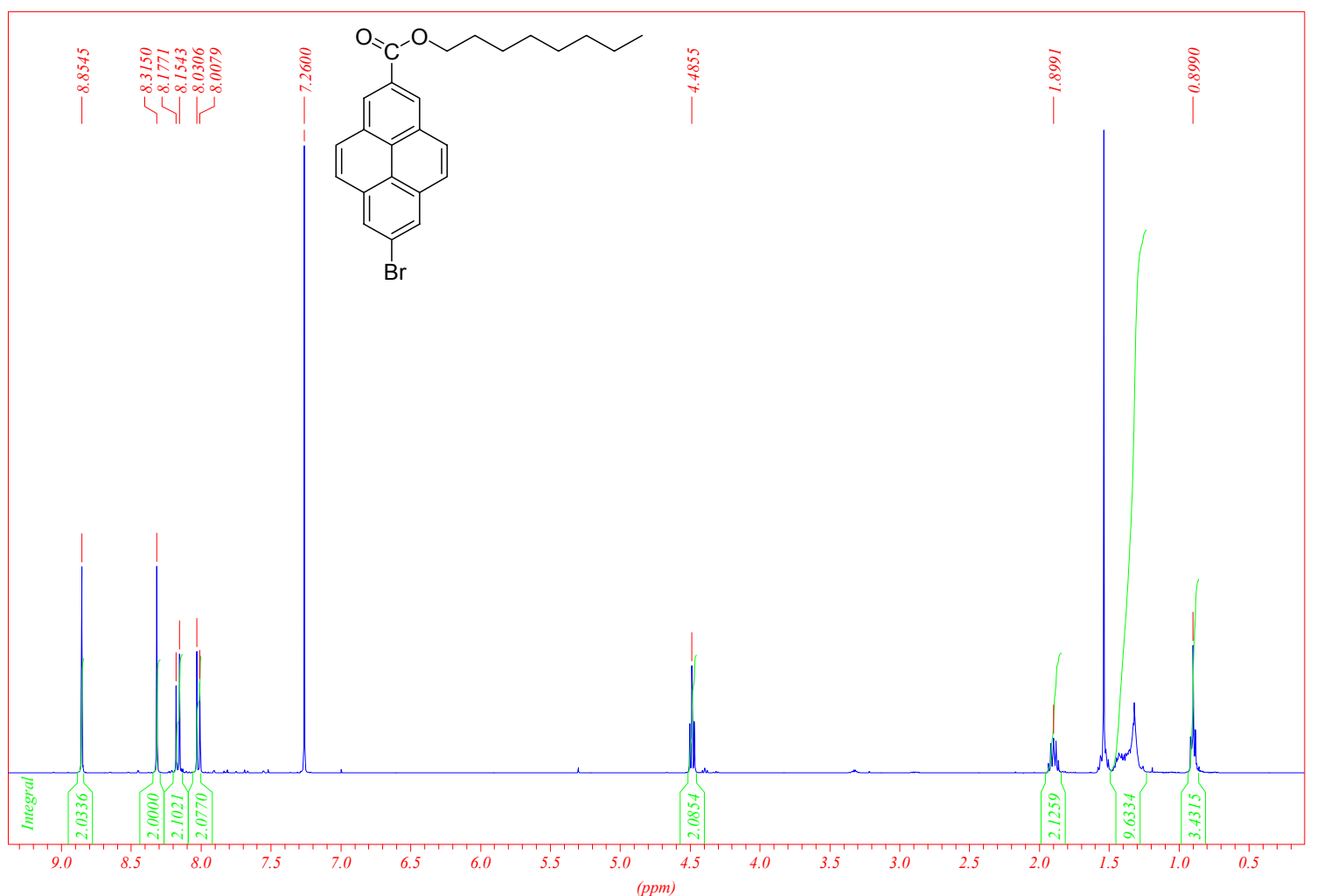

S- 9 
${ }^{13} \mathrm{C} \mathrm{NMR}\left(\mathrm{CDCl}_{3}\right)$ of 8

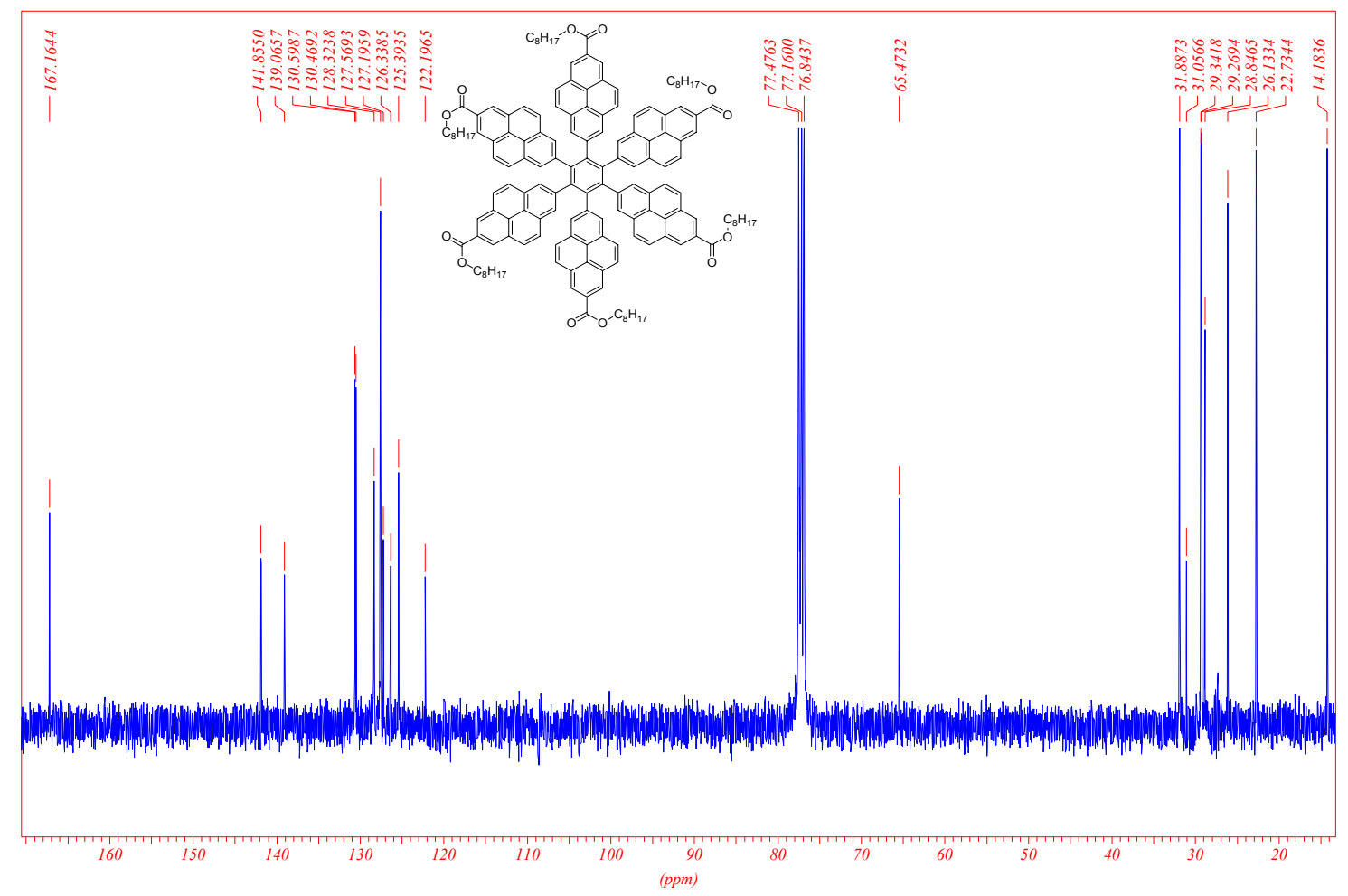

2) peak at 31.06 acetone $\mathrm{CH}_{3}$

\section{${ }^{1} \mathrm{H}$ NMR $\left(\mathrm{CDCl}_{3}\right)$ of 8}

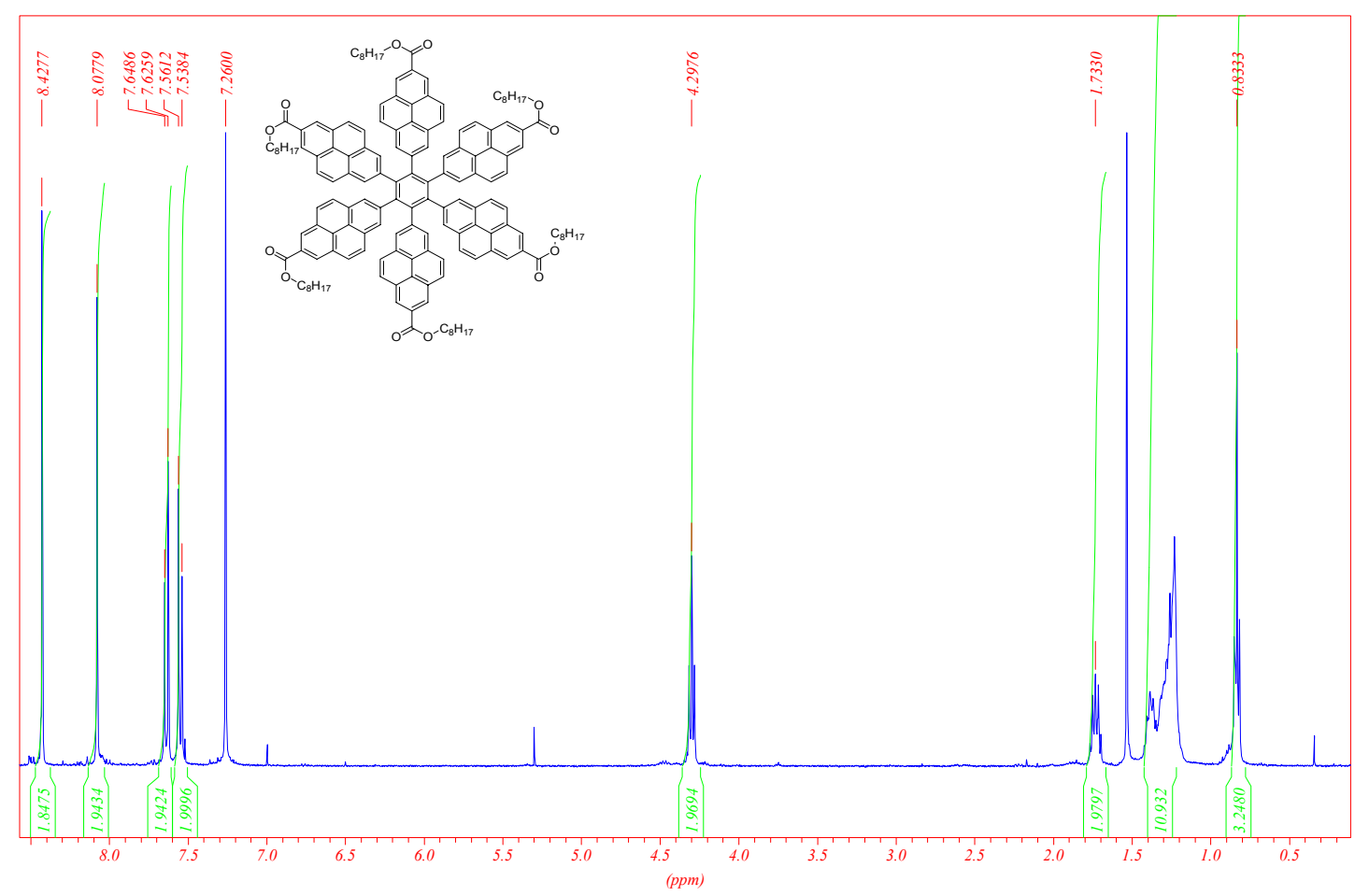

3) peak at 5.30 dichloromethane 
${ }^{1} \mathrm{H} \mathrm{NMR}\left(\mathrm{CDCl}_{3}\right)$ of $\mathbf{5}$

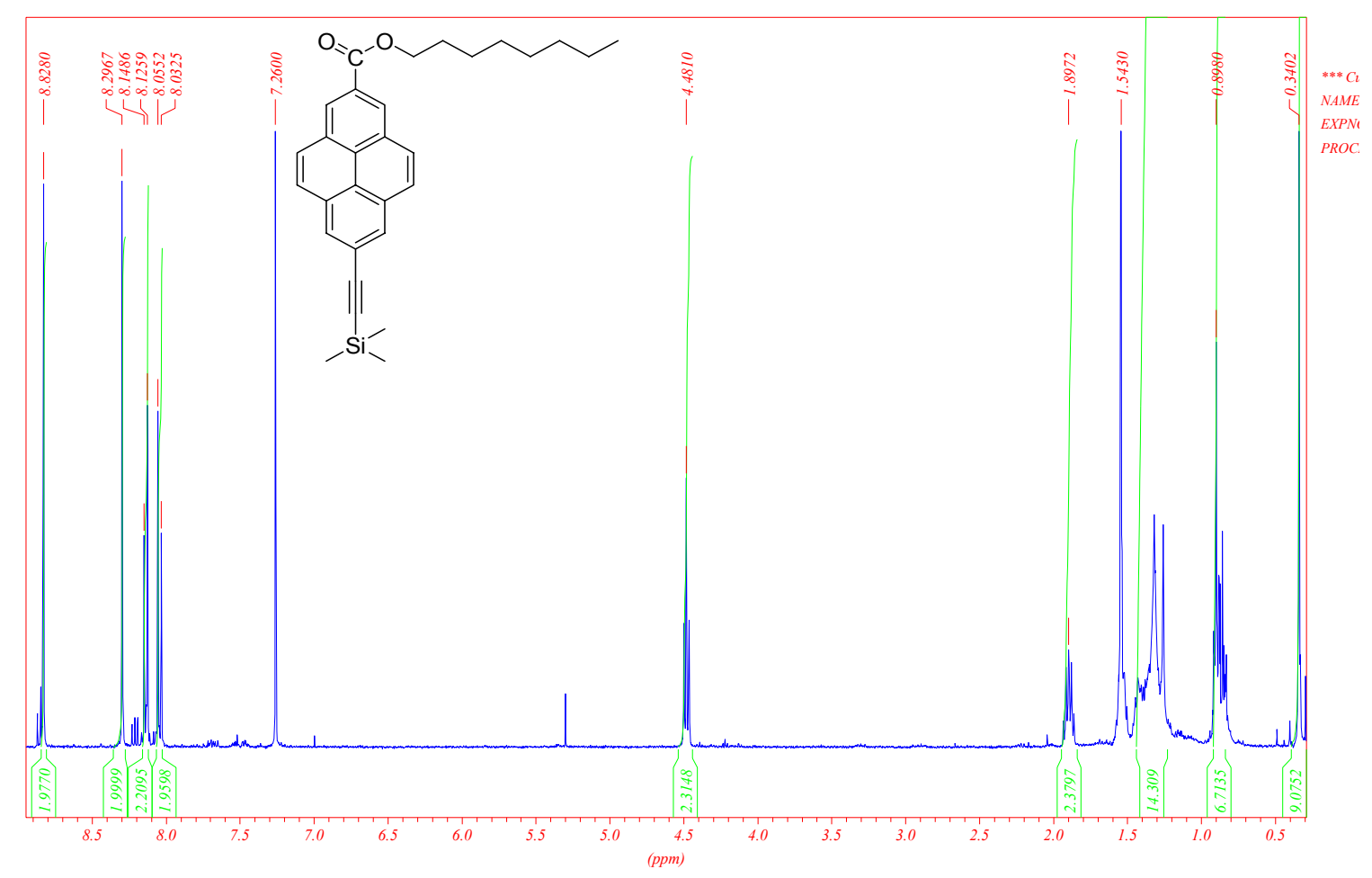

${ }^{1} \mathrm{H} \mathrm{NMR}\left(\mathrm{CDCl}_{3}\right)$ of 6

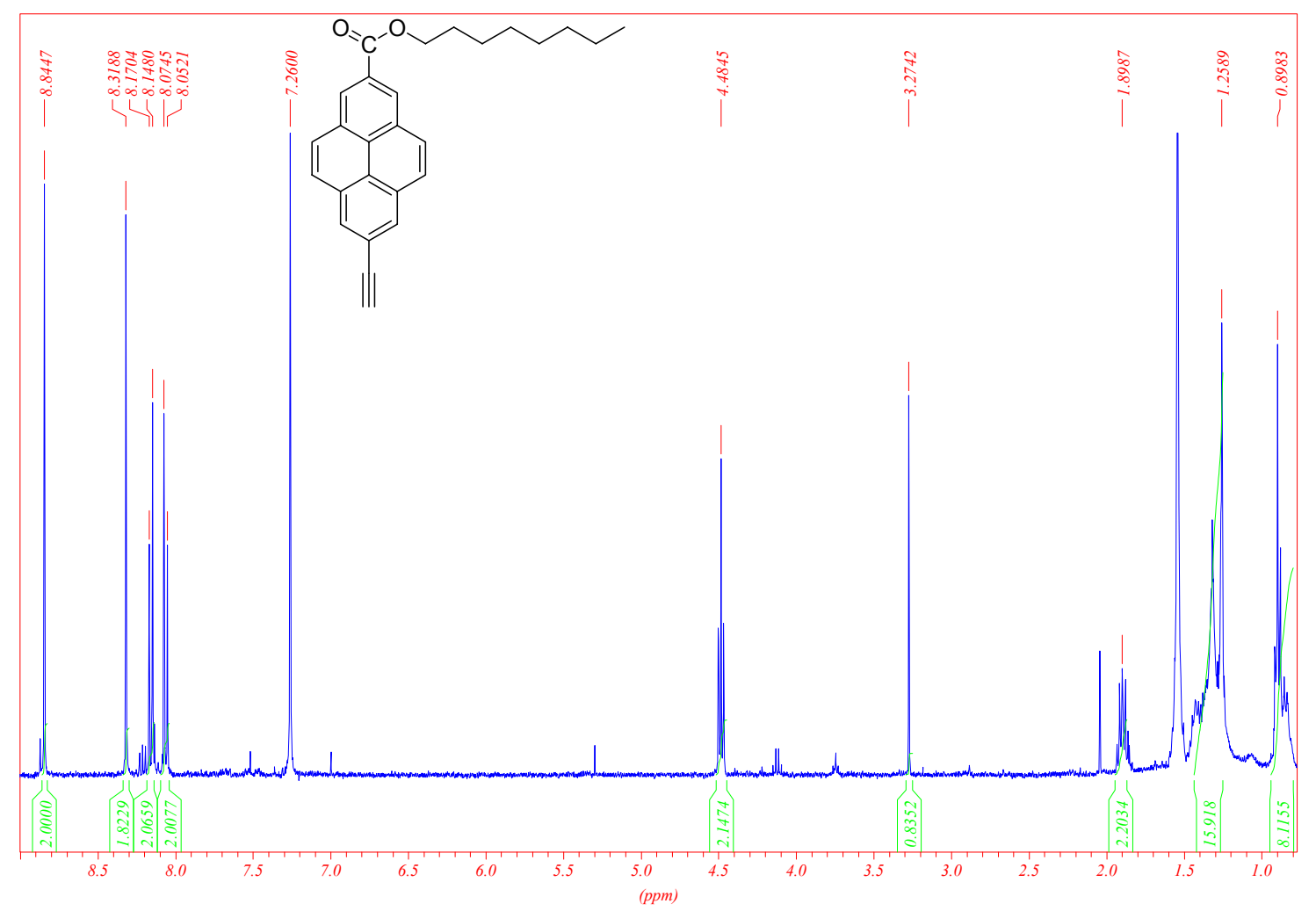

S- 11 
${ }^{1} \mathrm{H}$ NMR $\left(\mathrm{CDCl}_{3}\right)$ of 7

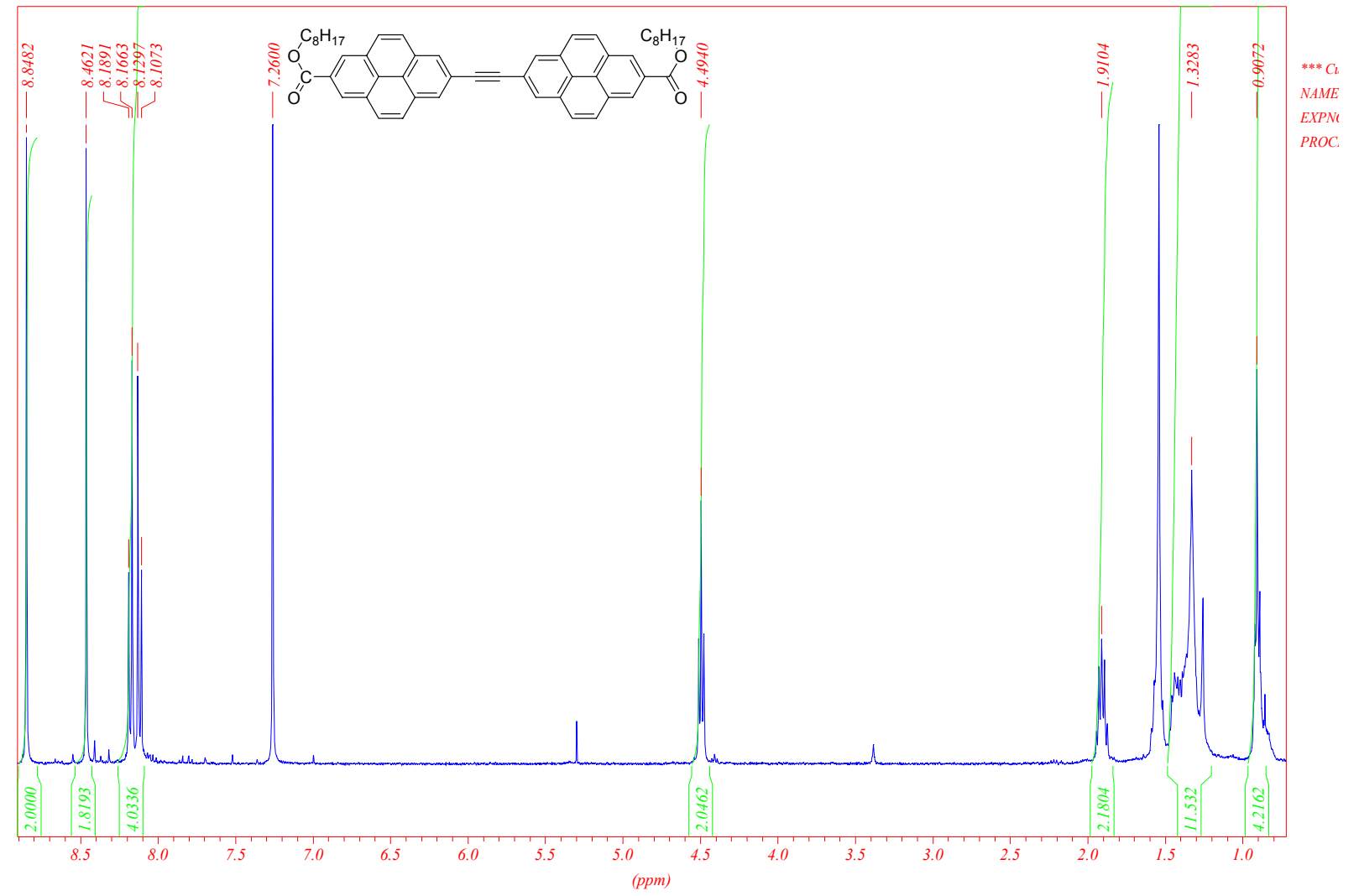

(1) Conner, M. D.; Scott, D. A.; Collard, M. D.; Liotta, L. C.; Schiraldi, D. A. J. Org. Chem. 1999, 64, 6888-6890. 